\title{
Feasibility Study of Nanocrystalline-Ribbon Cores for Polarized Inductive Power Transfer Pads
}

\author{
Daniel E. Gaona, Student Member, IEEE, Saikat Ghosh, Student Member, IEEE, \\ and Teng Long, Member, IEEE
}

\begin{abstract}
MnZn-based ferrite materials like the EPCOS N87 or K2004 are commonly used as magnetic cores in inductive power transfer (IPT) applications. However, the performance and the reliability of IPT systems are limited by ferrite's intrinsic brittleness and low flux density saturation point. In this paper, a study of nanocrystalline ribbon based magnetic cores for IPT applications is presented. FEM simulations and experimental validations are used to compare both materials. The design of ultra-thin laminated cores such as nanocrystalline ribbons for IPT systems is presented. Compared to ferrite, nanocrystalline ribbon is mechanically more robust; it has a higher magnetic permeability and a higher saturation point. Results show that nanocrystalline ribbon cores achieve more than a $50 \%$ volume reduction when used in IPT pads. This is due to nanocrystalline's high saturation point. However, a compromise arises as the total power loss increases due to the induced eddy currents in the core. The reduction of efficiency can be mitigated by special geometrical designs of the nanocrystalline ribbon cores. A $6.6 \mathrm{~kW}$ IPT system has been built for experimental validation of the design methodology.
\end{abstract}

Keywords-Inductive power transfer, nanocrystalline ribbon, magnetic materials, magnetic cores.

\section{INTRODUCTION}

In inductive power transfer (IPT) technology, AC magnetic fields are used to couple power between the transmitter and the receiver pads with large airgaps. For instance, airgaps between 10 and $20 \mathrm{~cm}$ are used in electric vehicle (EV) wireless charging. This magnetic field is produced by sets of copper coils carrying AC currents. An arrangement of magnetic cores is used to increase the flux density and channel the magnetic flux. Additionally, aluminum sheets are used as shields to prevent magnetic flux leakage to unwanted areas. For the coil, litz wire is used in order to reduce the skin and proximity effects which increase the $\mathrm{AC}$ resistance of the coil. For the core, MnZn based ferrite materials such as the EPCOS [1], [2] or K2004 [2], [3] are often used. However, the brittleness of these materials makes the IPT system prone to mechanical breakage and limits the implementation of large-scale and/or complex geometries. Moreover, ferrite cores have a lower flux density saturation point, which presents challenges for compact and high-power IPT systems. Hence, the power density of IPT charging pad is constrained by the core material.

In recent years, a lot of effort has been made to reduce the amount of the core material used in the pads as a way to increase their power density. In [4], the core bars were

This work was supported by the Engineering and Physical Sciences Research Council (EPSRC) (EP/R036799/1).

Daniel E. Gaona, Saikat Ghosh, and Teng Long are with the Department of Engineering, University of Cambridge, Cambridge, CB3 OFA, UK. (email: deg32@cam.ac.uk, ssg39@cam.ac.uk, t1322@cam.ac.uk (corresponding author: Teng Long)). reshaped in order to obtain a uniform flux density throughout the entire bar. On the other hand, in [5], an optimum 2$\mathrm{D}$ axisymmetric placement of the core for a polarized pad was studied. In both cases, optimum designs are intrinsically limited by ferrites poor mechanical (Young's modulus, yield strength, and manufacturability) and magnetic (magnetic saturation and permeability) properties [6], [7]. The need for new magnetic materials is evident. In [8], for instance, ferrite nano-particles on a base of polymer were introduced to improve the mechanical performance of the pads and reduce the amount ferrite. Although permeabilities are lower, the mechanical robustness of the material is increased. Iron and steel are not a desirable option due to their low permeability values. This is also true for powder-based cores. Permalloy, Sendust, and FE-based amorphous materials are other possible replacements for ferrite due to their higher saturation point. However, their saturation points and permeabilities are lower than that achievable by nanocrystalline cores [9].

Nanocrystalline materials, such as $\mathrm{FeCuNbSiB}$, present several advantages that makes them attractive for IPT applications [10]. First, compared to ferrite, nanocrystalline ribbons are mechanically more robust and can be formed into various geometries. This allows for a more robust, reliable, and flexible design. Second, it has a higher relative permeability $\mu_{r}$ which reach values up to 300000 [11], which is several orders of magnitude higher than that of ferrite. Higher $\mu_{r}$ constrains the magnetic flux more efficiently and reduces the flux leakage to shields or other metallic surfaces. As a result, the coupling coefficient $k$ can be potentially increased.

Nanocrystalline alloys saturate at $1.25 \mathrm{~T}$ which is more than twice the saturation point of ferrite. The higher the saturation flux density $B_{\text {sat }}$, the less core material is needed. Hence, more compact designs can be obtained. Moreover, Nanocrystalline's $B_{\text {sat }}$ is more constant with respect to temperature deviations as compared to ferrite. This is due to nanocrystalline's higher Curie temperature than that of ferrite. The gradual decay of $B_{\text {sat }}$ as a function of the temperature ensures satisfactory performance in the middle temperature range [10], [12]. As a result, nanocrystalline cores can operate at higher temperatures without compromising performance. Additionally, the requirements for cooling of the core can be relaxed which decreases the design complexity as well as pad's cost and size.

Nanocrystalline ribbon exhibits lower losses compared to ferrite when the flux is aligned with the ribbon orientation. The hysteresis loss is lower due to the soft magnetic characteristics of the nanocrystalline material. Eddy current loss is limited by using thin laminated ribbons. The typical thickness of nanocrystalline ribbon is between 14 to $30 \mu \mathrm{m}$ [9]. As the thickness of the ribbon decreases, so do the power losses. The eddy current loss, however, can become problematic when a component of the magnetic flux is perpendicular to the ribbon surface due to the high conductivity 


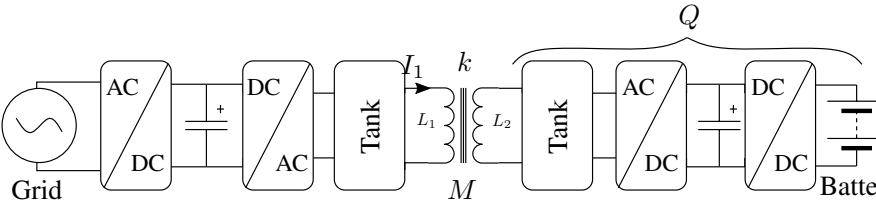

Fig. 1. Typical IPT structure. Tank: Compensation circuit (resonant tank). $M$ : Mutual inductance. $L_{1}$ : Transmitter inductance. $L_{2}$ : Receiver inductance. $k$ : Coupling factor. $Q$ : load quality factor $Q=R_{e q} / \omega L_{2}$ [1].

of the material: $\sigma=833333 \mathrm{~S} / \mathrm{m}$ [13]. This effect is yet to be evaluated for IPT applications.

This contribution evaluates the performance of nanocrystalline magnetic cores for IPT applications with the aid of FEM simulations and experimental set-ups. In section I, the simulation environment and comparison framework are presented. A methodology for simulating laminated cores is described. In section II, FEM simulations are used to determine the performance of nanocrystalline ribbon cores in IPT applications and their effect on the coupling coefficient $k$, flux distribution within the core bars, core losses, and power transfer capability of the pad. Section III covers the experimental validation of the results.

\section{Simulation EnVironment And Framework}

\section{A. Figure-of-Merit: IPT coil efficiency}

A simplified structure of an IPT system is shown in Fig. 1. At the first stage, the low-frequency AC voltage from the grid is rectified by a PFC circuit at the transmitter side. An inverter is used to excite the transmitter coil with high-frequency currents. Throughout this paper, a nominal frequency of $85 \mathrm{kHz}$ is used according to the standard SAE J2954 for electric vehicle applications. The induced voltage in the secondary is rectified to charge the battery. Compensation circuits (LCL, LCCL, C-series, or C-parallel) are needed for reactive power compensation and power flow control. In this context, the magnetic pads can be represented as a pair of loosely coupled inductors with different values of self $\left(L_{1}\right.$ and $L_{2}$ ) and mutual $(M)$ inductances. The coupling factor $k=M / \sqrt{L_{1} L_{2}}$ for such arrangements ranges between 0.15 and 0.5 depending on the magnetic design and the airgap between pads [14]. The mutual inductance and coupling factor are directly linked to the power transfer capability of the pad. The power transfer efficiency of magnetic coupler for a perfectly compensated system is given by (1) [15]:

$$
\eta_{\max }=\frac{k^{2} \cdot Q^{2}}{\left(1+\sqrt{1+k^{2} \cdot Q^{2}}\right)^{2}} \approx 1-\frac{2}{k Q}
$$

where $Q=\sqrt{Q_{1} Q_{2}}$ is the geometric mean of the quality factors of the transmitter and receiver coils respectively with $Q_{1}=\omega L_{1} / R_{1}$ and $Q_{2}=\omega L_{2} / R_{2} . R_{1}$ and $R_{2}$ refer to the equivalent resistance of the transmitter and receiver coils considering the cumulative effect of both copper and core losses.

The figure-of-merit of IPT pads efficiency is: $k Q$ [16]. Thus, for the same coupling factor $k$, pads with higher quality factors are more efficient. This figure-of-merit is used in this contribution when comparing ferrite and nanocrystalline ribbon cores.

\section{B. FEM Simulation}

Modeling the electromagnetic characteristics of IPT pads by means of FEM simulations is a common practice. The values of self and mutual inductances can be obtained with high accuracy by using FEM simulations. In this paper, the pads were simulated by using OCOMSOL. Simulation results were compared to previously reported values in [1], [2] matching with a difference of less than $5 \%$. The simulation results were also compared to experimental results (see Section V). with deviations of less than $8 \%$.

\section{Simulation of laminated nanocrystalline ribbon cores}

For ferrite (N87), the magnetic properties of the cores are homogeneous regardless of the orientation as the material is isotropic. However, for core bars made with nanocrystalline ribbons, each bar needs to be represented with anisotropic permeability and conductivity. The effect of laminations in numerical FEM simulation has been extensively researched since the last century by Steinmetz, Bertotti, and more recently by Hanhne et al., Bermudez [17] and Wang [18]. One of the most popular methods is the homogenization method since it permits to decrease the complexity of the FEM simulations with an acceptable compromise of the accuracy. Accuracies over $95 \%$ can be achieved as reported in [13]. This method uses the equivalent permeability $\left(\mu_{e q}\right)$ and conductivity $\left(\sigma_{e q}\right)$ for calculating magnetic flux, hysteresis losses, and eddy current losses [13].

A laminated core, shown in Fig. $2 a$ ), can be represented as a non-laminated one with equivalent conductivity $\sigma_{e q}$ and permeability $\mu_{e q}$ as shown in Fig. $2 b$ ). The equivalent parameters can be isotropic or anisotropic depending on the flux distribution. In transformers, for instance, the equivalent conductivity can be isotropic as the flux is mainly unidirectional. For cuboid-shaped magnetic cores in IPT applications, however, the flux paths are less constrained due to the large air gap in the magnetic path. Hence, the magnetic flux is multi-directional and, consequently, anisotropic properties must be considered. Several methods of calculating the equivalent properties for laminated cores are reported in literature such as the ones in [17], [18], [19], and [20]. They vary in terms of accuracy, assumptions, and complexity. In [17], an expression for the equivalent conductivity in the $y$ axis $\left(\sigma_{e q, y}\right)$, for a unity stacking factor $(F=1)$, is introduced as in $(2)$ :

$$
\sigma_{e q, y}=\sigma\left(\frac{T-2 \delta}{N(a+t-2 \delta)-a}\right)^{2}
$$

where $\sigma$ is the conductivity of the bulk material, $t$ and $a$ represent the ribbon thickness and width respectively (Fig.2),

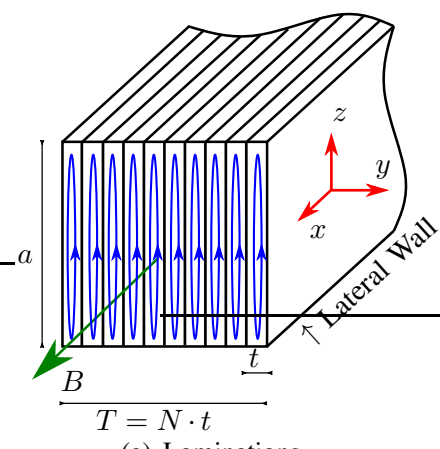

(a) Laminations

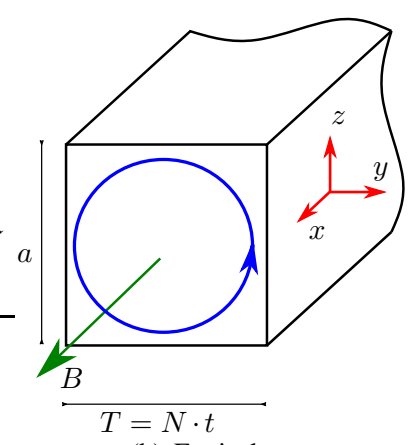

(b) Equivalent
Fig. 2. Depiction of a $a$ ) laminated core and its $b$ ) equivalent representation with a $100 \%$ stacking factor $F$ according to [17]. $T$ : Core bar width. $t$ : Ribbon thickness. $N$ : Number of ribbons per core bar. $a$ : Ribbon's width. $B$ : Magnetic flux. Spacing between lamination is not depicted. 
and $\delta$ is the skin-depth defined as $\delta=\left(\sqrt{\pi f \mu_{0} \mu_{r} \sigma}\right)^{-1}$ with $f$ as the frequency of operation $(85 \mathrm{kHz})$.

Equation (2) can be modified for the non-unity stacking factors (i.e. $F<1$ ) by dividing $\sigma_{e q, y}$ by $F$. The expression is close to the simplified expression introduced in [19]; thus, the simplified expression is adopted. The conductivities at different directions, considering the stacking factor, are shown in (3) [19]:

$$
\sigma_{e q, z}=\sigma_{e q, x}=F \sigma, \quad \sigma_{e q, y} \approx \frac{\sigma}{F}\left(\frac{t}{a}\right)^{2}
$$

The estimation of the equivalent anisotropic permeability is straightforward as only the stacking factor is considered as shown in (4) and (5) [13].

$$
\begin{gathered}
\mu_{e q, y}=\mu_{e q, z}=\frac{\mu \mu_{0}}{F \mu_{0}+(1-F) \mu} \\
\mu_{e q, x}=F \mu+(1-F) \mu_{0}
\end{gathered}
$$

Here, $\mu$ refers to the bulk permeability whilst $\mu_{0}$ refers to the permeability of free space. To facilitate the convergence of the model, linear magnetic properties are considered. The mesh is carefully selected to ensure the convergence of the FEA.

\section{Lamination orientation}

In power transformers, the flux path is unidirectional and the orientation of laminations is selected to facilitate their manufacturability. In IPT applications, however, the flux is multi-directional and, a priori, lamination orientation is not intuitive. The flux enters both the top and lateral faces of the core bars. However, as the majority of the flux enters the top face of the bar, the lamination orientation where the laminated thin ribbon is parallel to the major flux and perpendicular to the surface - as shown in Fig.2a) - was found to be the optimum.

\section{E. Power Loss Calculation}

1) Core Losses: At higher frequencies, power losses can be computed either using the concept of complex permeability or following the traditional Steinmetz method. The complex permeability model can be easily defined for isotropic materials but it complicates the FEA computations for anisotropic ones. Consequently, the Steinmetz method was used. This method is standard for core loss estimation in IPT designs as reported in [1]-[3], [15]. The power loss per unit volume is given by (6):

$$
P_{v}=C_{m} f^{\alpha} B^{\beta}
$$

where, $C_{m}, \alpha$, and $\beta$ are obtained from the data-sheets of each material. In principle, given the switching character of the power electronics, the Modified Steinmetz Equation (MSE) or the Generalized Steinmetz Equation (GSE) could be used to increase the accuracy. However, as long as a compensation circuit is in place, the current harmonics decay at $-40 \mathrm{~dB} / \mathrm{dec}$ which allows for the approximation of the current as a pure sinusoidal at $85 \mathrm{kHz}$. As a result, the standard Steinmetz equation is sufficient to estimate losses accurately: $P_{v}=C_{m}^{\prime} B^{\beta}$. The parameters for both materials are detailed in Table I.

From Table I, it is clear that nanocrystalline cores generate fewer losses than ferrite cores for the same non-traverse flux $B$. Traverse flux, however, inherently induces eddy
Table I. STEInMETZ PARAMETERS

\begin{tabular}{cc|cc} 
Material & Thickness & $C_{m}^{\prime}\left[\mathrm{W} / \mathrm{m}^{3} / \mathrm{T}^{\beta}\right]$ & $\beta$ \\
\hline \multirow{3}{*}{ Nanocrystalline } & $15 \mu \mathrm{m}$ & $2.27 \times 10^{6} \times F$ & 1.93 \\
& $20 \mu \mathrm{m}$ & $3.01 \times 10^{6} \times F$ & 1.93 \\
& $25 \mu \mathrm{m}$ & $4.48 \times 10^{6} \times F$ & 1.93 \\
\hline N87 & $2.5 \times 10^{7}$ & 2.7 \\
\hline \multicolumn{4}{r}{$F:$ Stacking Factor }
\end{tabular}

currents in the laminations which in turn increases the overall power losses. In fact, even in nanocrystalline Ushaped inductors with small air gaps, gap losses can account for up to $40 \%$ of the total losses at high flux densities [13]. In [13], the estimation of losses via FEM simulation by means of the homogenized method (see Section II-C) was successfully validated with an accuracy of $95 \%$. Using the same approach, the eddy current losses and the hysteresis losses, hereafter referred to as $P_{\text {elec }}$ and $P_{\text {mag }}$ respectively, can be estimated for nanocrystalline ribbon cores.

2) Copper losses: For the copper losses, the method presented in [15] was used. These losses are of two natures: skin effect (including DC losses) and the proximity effect.

a) Skin Effect: The skin effect losses are due to the AC current through each conductor. The self-induced eddy current losses change the distribution of the current in its cross-sectional area. In a litz wire, the skin effect losses are reduced due to the minimum radius of the strands. However, they are not entirely eliminated. An estimation of such losses, per unit length, is given by (7) [21]:

$$
P_{s k i n, L}=n \cdot R_{D C} \cdot F_{R}(f) \cdot\left(\frac{i_{p k}}{n}\right)^{2} \quad[W / m]
$$

Here, $n$ is the number of strands, $r_{i}$ is the strand radius, $R_{D C}=\left(\sigma \pi r_{i}^{2}\right)^{-1}$, and $F_{R}(f)$ represent the increment of the resistance with the operating frequency as shown in (8) [21]:

$$
\begin{array}{r}
F_{R}=\frac{\xi}{4 \sqrt{2}}\left(\frac{\operatorname{ber}_{0}(\xi) \operatorname{bei}_{1}(\xi)-\operatorname{ber}_{0}(\xi) \operatorname{ber}_{1}(\xi)}{\operatorname{ber}_{1}(\xi)^{2}+\operatorname{bei}_{1}(\xi)^{2}}\right. \\
\left.-\frac{\operatorname{bei}_{0}(\xi) \operatorname{ber}_{1}(\xi)+\operatorname{bei}_{0}(\xi) \operatorname{bei}_{1}(\xi)}{\operatorname{ber}_{1}(\xi)^{2}+\operatorname{bei}_{1}(\xi)^{2}}\right)
\end{array}
$$

where $\xi$ represents the ratio between the skin depth $\delta_{c u}$ of copper to the strand diameter:

$$
\xi=2 \cdot r_{i} /\left(\sqrt{2} \delta_{c u}\right) \quad \text { with } \quad \delta_{c u}=\frac{1}{\sqrt{\pi \mu_{0} \sigma f}}
$$

Likewise, ber $_{x}$ and bei $_{x}$ correspond to real and imaginary part of the Kelvin functions of order $x$. The Kelvin functions are the solution to the $x^{t h}$ order Bessel solution of the first kind $J_{x}(z)$ when the argument $z$ is considered as real. In other words:

$$
\operatorname{ber}_{x}(\xi)=\Re\left(J_{x}\left(\xi e^{j \frac{3 \cdot \pi}{4}}\right)\right)
$$

b) Proximity Effect: Proximity effect losses are a consequence of the alternative fields $H$ perpendicular to the coil. The magnetic field that leads to proximity losses results from the sum of the external field $H_{e}$ and the internal field $H_{i}$. The former is the field induced by neighboring conductors. On the contrary, $H_{i}$ in one strand originates from its neighboring strands. The internal $H_{i}$ can be approximated by (11) [21]: 


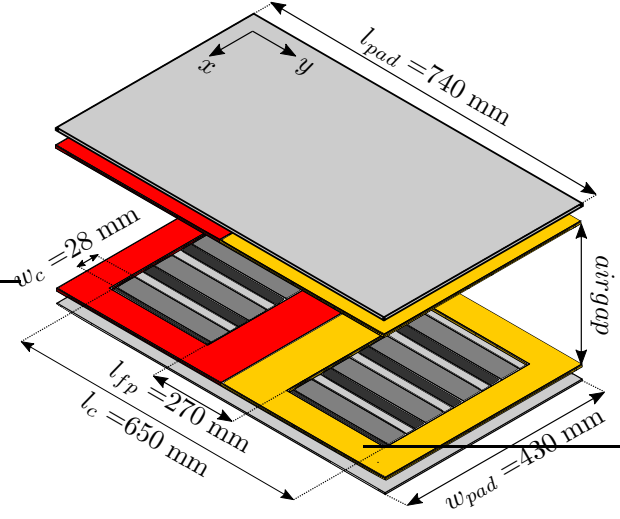

(a) Double-D pad

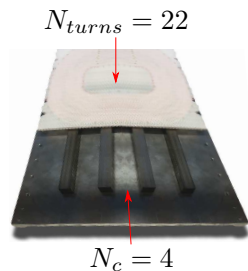

(b)

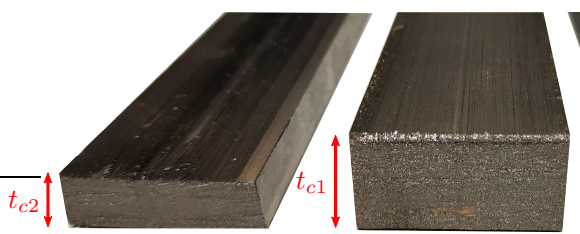

(c)

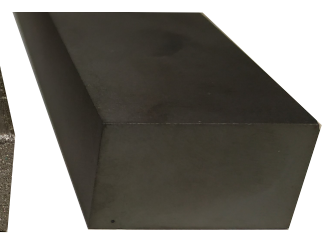

Fig. 3. a) Double-D Pad's Dimensions. b) Depiction of the Double-D pad used in this paper. c) From left to right, Nanocrystalline core $t_{c 2}=$ $8 \mathrm{~mm}$, Nanocrystalline core $t_{c 1}=16 \mathrm{~mm}$, ferrite core $t_{f e}=t_{c 1}$.

$$
H_{i}=\frac{i_{p k}}{2 \cdot \sqrt{2} \cdot \pi \cdot r_{c}}
$$

where $r_{c}$ is the litz wire radius. Consequently, the total proximity losses can be calculated by (12):

$$
\begin{aligned}
P_{\text {prox }, L} & =P_{\text {prox }, L, e}+P_{\text {prox }, L, i}[W / m] \\
& =n \cdot R_{D C} \cdot G_{R}(f)\left(H_{e, p k}+\frac{i_{p k}^{2}}{8 \cdot \pi^{2} \cdot r_{c}^{2}}\right)
\end{aligned}
$$

where, $G_{R}$ defines the increment of the proximity effect as a function of the frequency and it can be calculated as in (13) $[21]:$

$$
\begin{array}{r}
G_{R}=-\frac{\xi \pi^{2} d_{i}^{2}}{2 \sqrt{2}}\left(\frac{\operatorname{ber}_{2}(\xi) \operatorname{ber}_{1}(\xi)+\text { ber }_{2}(\xi) \text { bei }_{1}(\xi)}{\operatorname{ber}_{0}(\xi)^{2}+\text { bei }_{0}(\xi)^{2}}\right. \\
\left.+\frac{\text { bei }_{2}(\xi) \operatorname{bei}_{1}(\xi)-\operatorname{bei}_{2}(\xi) \operatorname{ber}_{1}(\xi)}{\operatorname{ber}_{0}(\xi)^{2}+\operatorname{bei}_{0}(\xi)^{2}}\right)
\end{array}
$$

Here, the estimation of $H_{e}$ is done analytically as in [2]. A different value of $H_{e}$ is obtained for each section of the coil depending on its position with respect to the core. A detail explanation of the methodology can be found in [2] and [21].

3) Compensation Capacitor Losses: The losses in the compensation capacitors can be calculated from (14):

$$
P_{L, \text { cap }}=\frac{\tan \delta\left(f_{0}\right)}{4 \cdot \pi f_{0} C} \cdot i_{p k}^{2}, \quad f_{0}=85 \mathrm{kHz}
$$

Here, $C$ corresponds to the capacitance value while $\delta\left(f_{0}\right)=$ 1.85 is the dissipation factor. The latter is identified from the datasheet of the TDK's Metallized Polypropylene Film Capacitors (MKP) Series B32651.

\section{F. Inductive couplers}

Two identical Double-D pads were manufactured to validate the simulation results: one with nanocrystalline ribbon cores and the other with N87 cores. Pad dimensions are shown in Fig. 3. The dimensions are based on a previous publication [22] in order to have a reference for comparison.

The coil consists of 20 turns of litz wire which is made of 850 strands of $0.1 \mathrm{~mm}$ diameter, giving a total crosssectional area of $5.5 \mathrm{~mm}^{2}$. Four rectangular bars form the core. For the ferrite pad, each core bar is made of 7 I93 $(93 \times 28 \times 16 \mathrm{~mm})$ cores from EPCOS. The cores occupy approximately $30 \%$ of the pad area. The $16 \mathrm{~mm}$ core thickness ensures maximum flux values of $350 \mathrm{mT}$ during operation.

For the nanocrystalline cores, each core bar is a single unit. Each bar is formed by $20 \mu \mathrm{m}$ laminated ribbons with a stacking factor of 0.78 . Each ribbon is insulated from each other. Based on the aforementioned orientation of the laminations, the width of the ribbon determines the thickness of the bar. Ribbons of two different widths have been used to realize two different core thicknesses: $t_{c 1}=16 \mathrm{~mm}$ and $t_{c 2}=8 \mathrm{~mm}$.

\section{COMPARISON OF SELF $L_{1}$ AND MUTUAL $M$ INDUCTANCES}

The magnetic and electric properties of the materials have a direct impact on the values of self and mutual inductance of an IPT pad. The effect of the material permeability and conductivity are analyzed in the following sections.

\section{A. Effect of core permeability on self $L_{1}$ and mutual $M$ inductances}

Fig. 4 compares the self-inductance $L_{1}$, mutual inductance $M$, and coupling factor $k$ when using ferrite and nanocrystalline cores respectively. The coil at the transmitter side is identical to that at the receiver side; thus, the self-inductance at each coil is identical, i.e. $L_{1}=L_{2}=L$. First, the materials are simulated as lossless (i.e. $\sigma=0$ ) to isolate the effect of higher permeability. Given that the mutual inductance is inversely proportional to the magnetic reluctance $\Re$, the higher magnetic permeability of the nanocrystalline ribbon is expected to decrease $\Re$ and yield higher values of $L$ and $M$. In fact, a small increment of $L, k$, and $M$ is observed when using nanocrystalline ribbon cores as compared to ferrite cores. An increment of only $5 \%$ to $9 \%$ (depending on the airgap) was observed despite the fact that the nanocrystalline ribbon's permeability is more than 100 times higher than that of the ferrite N87 used in this comparison,

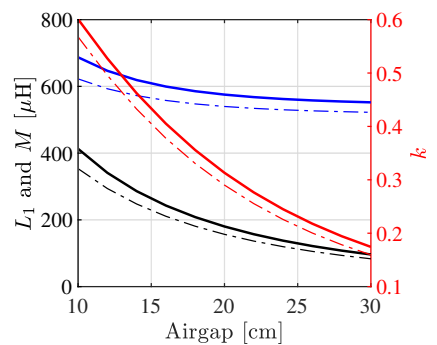

(a)

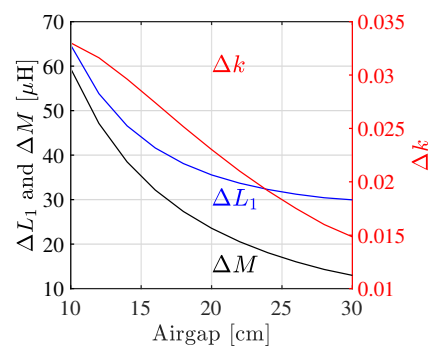

(b)
Fig. 4. a) Coupling factor $k$, self $L$, and mutual $M$ inductances for nanocrystalline ribbon (-) and ferrite N87 (.-) cores with $\sigma_{x, y, z}=0$. b) Parameter difference between nanocrystalline ribbon and ferrite cores: $\Delta=X_{\text {nano }}-X_{N 87}$, where $X$ is $k, L_{1}$, and $M$ respectively. 


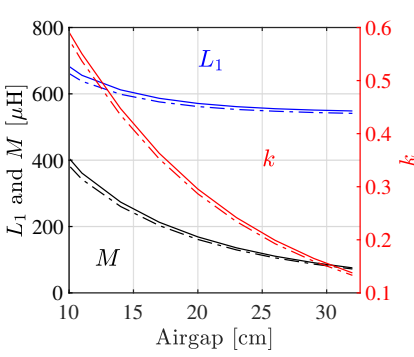

(a)

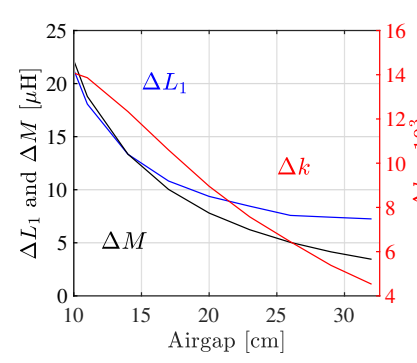

(b)
Fig. 5. a) Coupling factor $k$, self $L$, and mutual $M$ inductances for nanocrystalline ribbon (-) and ferrite N87 (.-) cores with $\sigma_{x, y, z} \neq 0$. b) Parameter difference between nanocrystalline ribbon and ferrite cores: $\Delta=X_{\text {nano }}-X_{N 87}$, where $X$ is $k, L_{1}$, and $M$ respectively.

The reason for such a small increment is that the airgap between pads dominates the magnetic circuit. The lower core reluctance has an insignificant effect on the overall magnetic flux. Thus, the benefits of higher permeability are trivial.

\section{B. Effect of core conductivity on self $L_{1}$ and mutual $M$ inductances}

For ferrite, the eddy currents induced in the core are very small due to the high resistivity $(10 \Omega \mathrm{m})$ of the material. Thus, the effect of eddy currents is almost neg- ligible. However, the resistivity of nanocrystalline material $\left(1.2 \times 10^{-6} \Omega \mathrm{m}\right)$, is considerably lower than that of ferrite. Due to the higher conductivity, eddy currents are induced in the cores causing changes of the overall coil inductance. As seen in Fig.5a) - b), the self and mutual inductances obtained from nanocrystalline cores are $1.5 \%$ to $3 \%$ higher than that of ferrite cores. The increment on the inductance obtained due to the higher permeability is counterbalanced by the induced eddy current in the nanocrystalline cores.

Core bars, as opposed to plates, are commonly used to constrain effectively the magnetic flux while reducing magnetic material, cost, weight, and fragility [22]. However, a considerable portion of the flux enters through the lateral faces of the bars and, consequently, the effect of the induced eddy-currents is not negligible. The overall effect will depend on the exact design of the pad; i.e., number of cores, core dimensions, etc. For smaller pads, for instance, the reduction of the magnetic performance due to eddy currents outweighs the improvement expected from the higher permeability. The variation of the magnetic performance of the pad is insignificant; i.e., replacing nanocrystalline cores for ferrite cores has a negligible effect on $k, L$, or $M$.

\section{Experimental Results}

The impedance measurements for the three aforementioned pads are shown in Fig. $6 a)-c$ ) at different airgaps

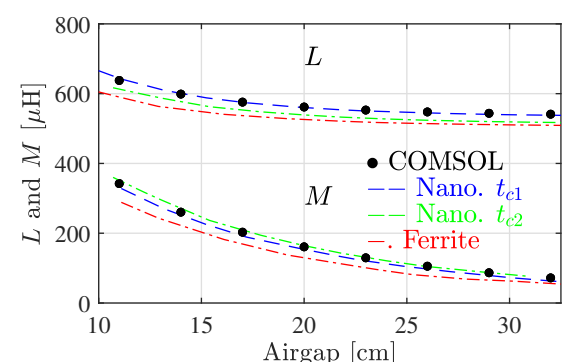

(a)

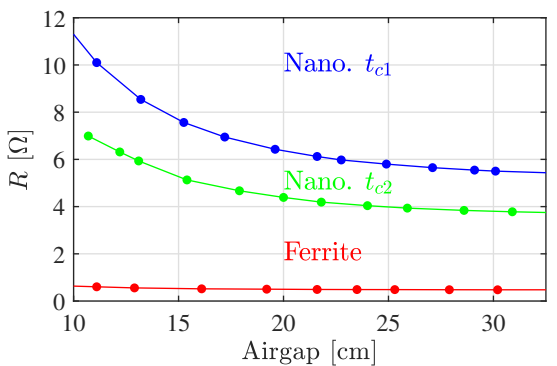

(d)

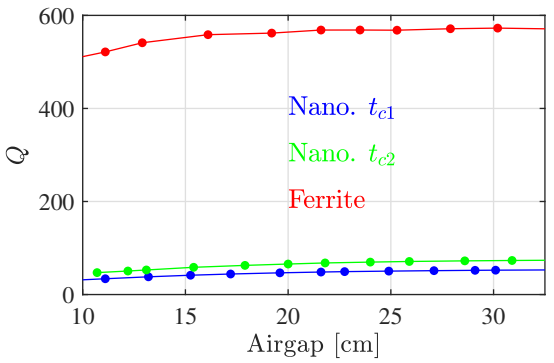

(g)

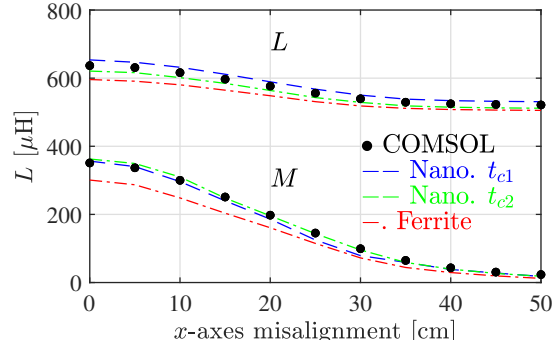

(b)

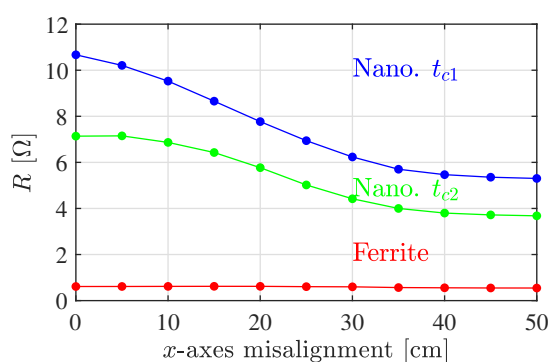

(e)

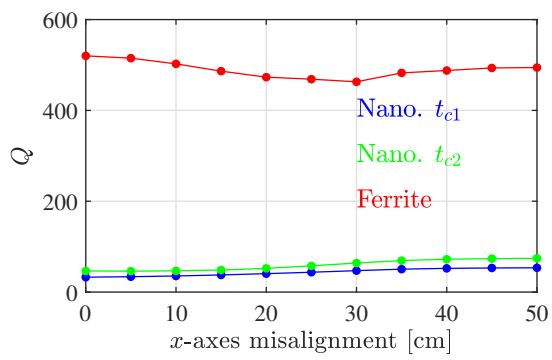

(h)

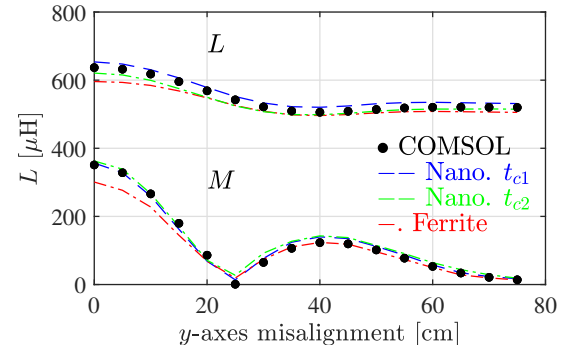

(c)

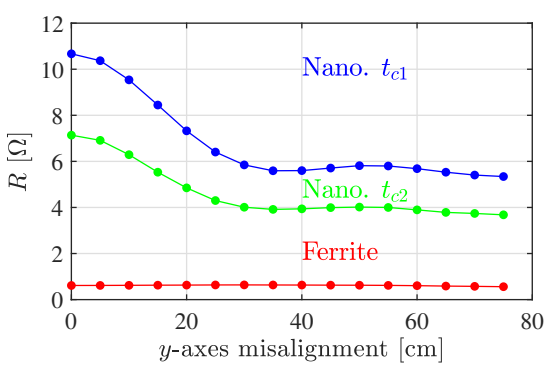

(f)

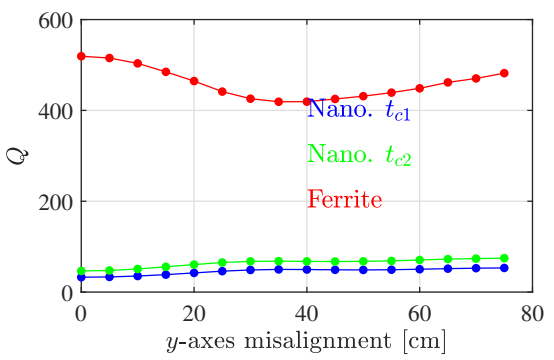

(i)

Fig. 6. Measurement of inductances, equivalent resistance, and quality factor of a Double-D pad with nanocrystalline and N87 cores at different pad misalignments. $a)-c$ ) Measured self $L_{1}=L_{2}=L$ and mutual $M$ inductances. $\left.d\right)-f$ ) Measured pad's AC equivalent resistance $R$ at $\left.85 \mathrm{kHz} . f\right)-i$ ) Coil quality factor $Q=\omega L_{1} / R . t_{c 1}=16 \mathrm{~mm} . t_{c 2}=8 \mathrm{~mm}$. COMSOL is the FEM estimation of the nanocrystalline $t_{c 1}$. 


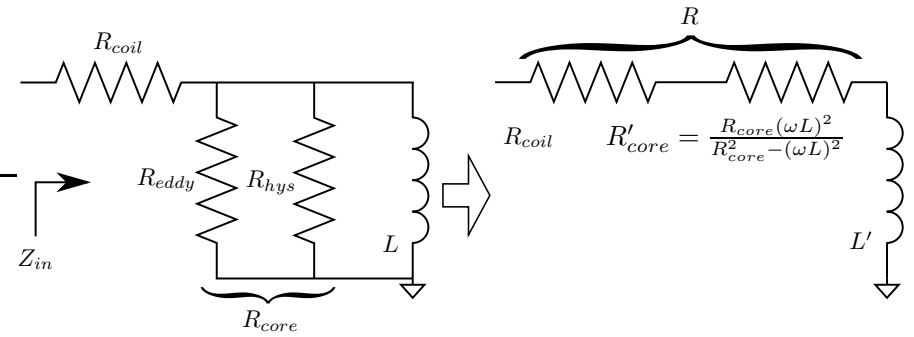

Fig. 7. Equivalent circuit of the magnetic coupler. On the left, a traditional model of the eddy current and hysteresis losses in the core as parallel resistors, $R_{e d d y}$ and $R_{h y s}$ respectively, is depicted. On the right, the equivalent circuit is considered as a series impedance. $L^{\prime} \approx L$ as $R_{\text {core }}$ is large.

and pad misalignments. They are measured at $85 \mathrm{kHz}$ using the impedance analyzer N4L PSM3750.

The inductance values match the FEM simulations with a difference of less than $8 \%$. Both nanocrystalline pads yield slightly higher self-inductance values than that of ferrite. This is attributed to their higher relative permeability, $\mu_{r}$. For both nanocrystalline pads, the mutual inductance remains fairly constant and it is higher than the one obtained from ferrite cores.

Fig.6d) - f ) depicts the equivalent resistance of the pad measured with the impedance analyzer. The equivalent impedance considers a series-connected resistor and inductor as shown in Fig.7. The equivalent resistance $R$ consists of both the high-frequency ohmic resistance of the coil (litz wire) and the core losses which are part of the real component of the complex impedance of the magnetic core. Conventionally, the equivalent circuit model of magnetic couplers uses resistors in parallel with the magnetizing inductor to model the eddy current and hysteresis losses as shown in Fig.7. The value of the resistance that represents the eddy current loss is inversely proportional to the conductivity of the magnetic core; i.e., higher conductivity results in lower eddy current resistance and thus higher eddy current loss. In this paper, the loss is modeled by a resistor in series with the magnetizing inductor. When using the series model, higher core conductivity results in a larger eddy current resistance in the equivalent circuit. The DC resistance of the coil was measured as $148 \mathrm{~m} \Omega$. For nanocrystalline pads, the resistance has been found to be between 7.6 and 20 times larger than that of ferrite's pads depending on the pad misalignment and the core thickness. The pad with slimmer nanocrystalline cores yields lower resistance. As the core thickness reduces, the eddy currents in the lateral faces decrease proportionally.

Nanocrystalline's higher equivalent resistance reduces the effective pad's quality factor $Q$ as shown in Fig. $6 g)-i$ ). While $Q$ ranges from $500-570$ for the ferrite pad, it reaches a maximum value of 50 and 75 for the nanocrystalline pads with $t_{c 1}$ and $t_{c 2}$, respectively. As discussed in Section II, the efficiency of the pad is proportional to $Q$. Hence, pads with nanocrystalline cores are expected to have a lower efficiency as compared to pads with ferrite cores. This is analyzed with more detail in the next section.

\section{COMPARISON OF FLUX DistRIBUTION WITHIN THE CORE}

The anisotropic characteristic of the permeability along with the eddy currents induced in the magnetic cores influences the distribution of magnetic flux within the core. A comparison of the flux distribution in the core is shown in Fig. 8 for N87 and nanocrystalline ribbon materials.

1) Ferrite Cores: Due to the isotropic properties of the material, the flux distribution in the cross-sectional area of the N87 cores is practically uniform as depicted in Fig. 8a). The flux density reaches its maximum value at the center of the coil since that section offers a shorter flux path between two coils; i.e, nearly all flux paths cross the center of the core. The flux density value is directly proportional to the operating current and inversely proportional to the air-gap. For large airgaps $(\geq 50 \mathrm{~mm})$, the leakage flux accounts for most of the linkage flux. Here, the leakage flux is defined as the portion of flux linking only the transmitter or receiver pads but not both. Therefore, the flux density remains fairly constant, as seen in Fig. 8c), and the self-inductance $L_{1}$ only shows a negligible change. Once the operating point (airgap and current value) is decided, the thickness of the ferrite core can be selected accordingly to ensure that saturation does not occur at any point within the core.

2) Nanocrystalline Cores: As opposed to the ferrite core, the flux distribution in the cross-sectional area of the nanocrystalline core is not uniform. Due to the large discrepancy between the permeability at the parallel and perpendicular directions with respect to the ribbon, the parallel direction ( $x$-axis) is the preferred magnetic path for the flux. The perpendicular direction ( $y$-axis) represents a high reluctance path due to the binder between ribbons. Thus, the flux only reaches a ribbon once the prevailing ones are close to saturation. As a result, the core lateral faces show the highest flux density. From the lateral face to the middle of the core bar ( $y$-axis), the flux density decreases. The middle section of the core bars ( $x$-axis) shows larger flux density than that at the ends, similar to ferrite case. A model of this effect is shown in Fig.9.

Here, $\psi$ represents the total flux entering the lateral faces of the core bar. Since the reluctance $\Re>>r$, the first lamination will uptake most of $\psi$ until it reaches saturation.

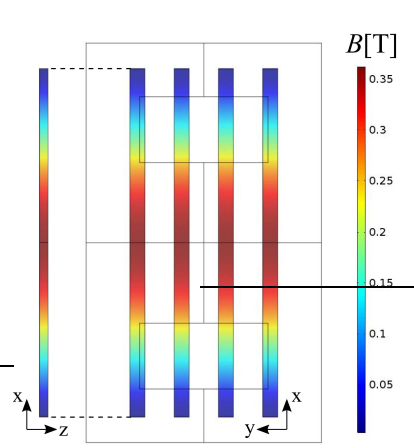

(a) N87

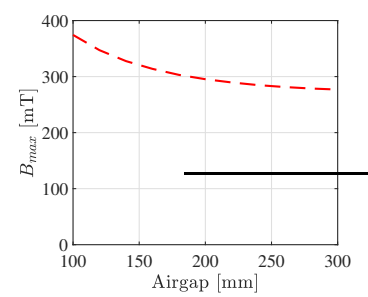

(c) N87

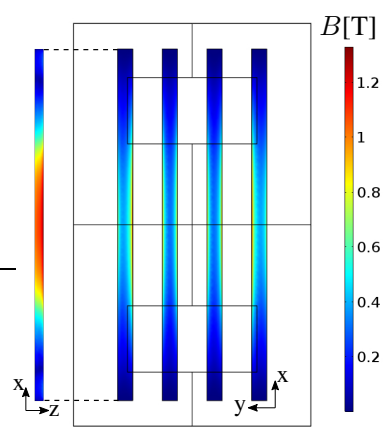

(b) Nanocrystalline $\sigma_{e q}=0$

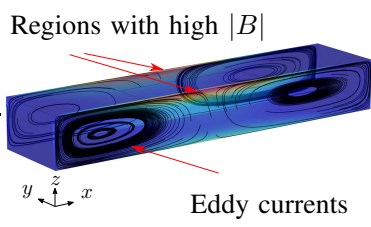

(d) Nanocrystalline $\sigma_{e q} \neq 0$
Fig. 8. Flux density distribution for $a$ ) N87 b) nanocrystalline cores at an airgap of $10 \mathrm{~cm} . c$ ) Flux density in the middle of the ferrite core as a function of the airgap. $d$ ) Eddy currents induced in the nanocrystalline core. Result obtained from pad shown in Fig.3. 


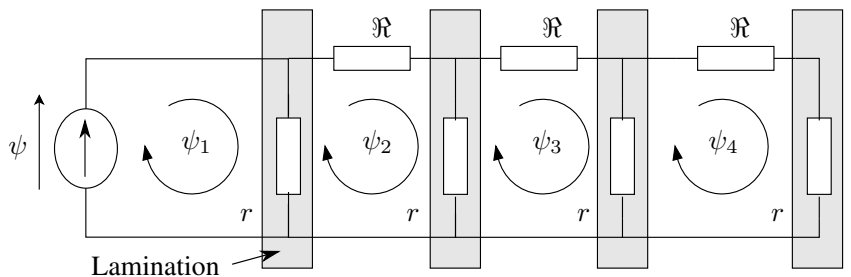

Fig. 9. Representation of the traverse flux magnetic $\psi$ entering the lateral faces of the cuboid core bars. $r$ and $\Re$ represent the magnetic reluctance of the nanocrystalline ribbon and binder between ribbons, respectively. $\Re>>$ r. $\psi_{1}>>\psi_{2}>>\psi_{3}>>\psi_{4}$

The subsequent ribbons will carry decreasing amounts of flux. The saturated region represents only a small portion of the total core volume as seen in Fig. $8 b$ ). The higher flux density at the lateral faces implies higher hysteresis losses. Additionally, the flux perpendicular to the ribbons induces eddy currents in the lateral faces of the core which changes the distribution and increases the power losses. These losses have a detrimental effect on the overall efficiency of the pad. Nevertheless, it is important to note that only a small portion of the core bar saturates. In fact, the flux density in the majority of the bar is similar to that of obtained with ferrite cores and therefore far from the saturation point of the nanocrystalline material. This suggests that the thickness of the bar can be further reduced resulting in higher power densities.

\section{EXPerimental Measurement of Power Losses AND COMPARISON}

To measure the power losses of the pad during normal operation, the set-up in Fig.10 is used. Series compensation circuits are used for simplicity in both transmitter and receiver sides. The resonant circuit is tuned for an airgap of $20 \mathrm{~cm}$ and perfect alignment between the transmitter and the receiver pads. At these conditions, $L_{1}=515 \mu \mathrm{H}$, $M=130 \mu \mathrm{H}$, and $C_{S}=1 /\left(\omega^{2} L_{1}\right)=6.8 \mathrm{nF}$. The latter is achieved by connecting ten TDK capacitors of $68 \mathrm{nF}$ in series. The inverter at the transmitter side uses a SiC MOSFETbased (CREE, C3M0075120K) H-Bridge. The rectifier at the receiver uses a $\mathrm{SiC}$ diode (On Semiconductor, 512FFSH40120ADNF155) bridge. A Texas Instrument DSP

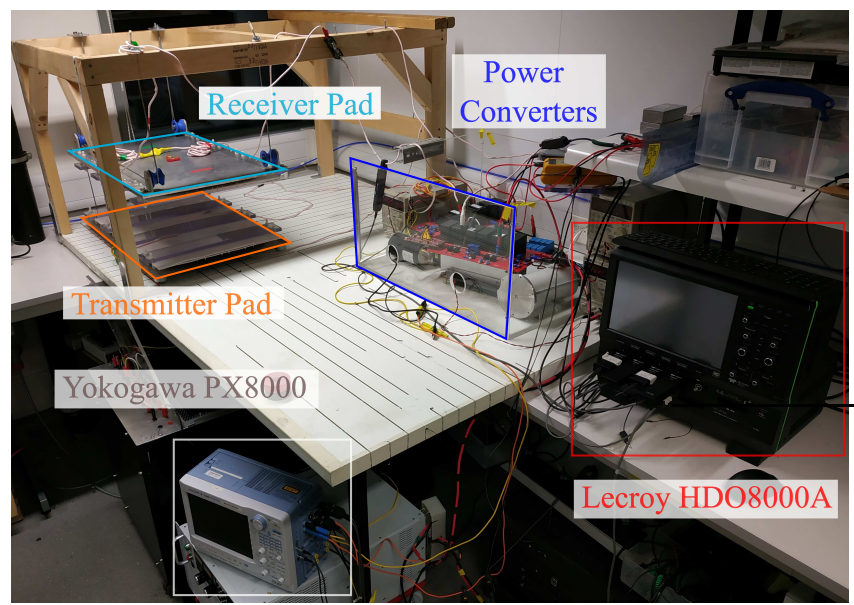

Fig. 10. Illustration of the testbench used for the experimental validation. The primary converter is a SiC MOSFET-based H-bridge. The secondary converter is a $\mathrm{SiC}$ diode bridge rectifier. controller (LAUNCHXL-F28379D) is used for generating the switching signals.

It is well known that the efficiency of the pad depends on the load [23]. In the case of identical transmitter and receiver pads, the maximum efficiency of the system is achieved when the currents in both coils are equal. This operating point occurs when the equivalent AC load on the receiver side is $R_{L, a c}=\omega M=69.4 \Omega$. This AC resistance is equivalent to a DC resistance of approximately $85 \Omega$ after the receiver rectifier stage. The maximum efficiency point is independent of the voltage magnitude [15]. Therefore, the power transfer can be regulated by adjusting the input DC-link voltage while maintaining maximum operational efficiency.

Two meters are used to estimate the DC and AC power respectively. A LeCroy HDO8000A is used to acquire AC waveforms and measured AC power. Fig.11 shows the voltage and current waveforms at the converter terminals for an operating point of $5 \mathrm{~kW}$. A Yokogawa PX8000 is used at the DC sides to measure the overall system efficiency. The power and efficiency measurements are shown in Fig.12 for the different pads.

Fig.12 shows the power input of both pads, the power losses in the magnetic coupler as well as the pad and system efficiencies respectively. The lower efficiency of the nanocrystalline pad is evident as shown in Fig.12d). At about $5 \mathrm{~kW}$, the power losses in the ferrite and nanocrystalline pad are approximately $200 \mathrm{~W}$ and $600 \mathrm{~W}$, respectively. The pad efficiency is approximately $88 \%$ for nanocrystalline pad

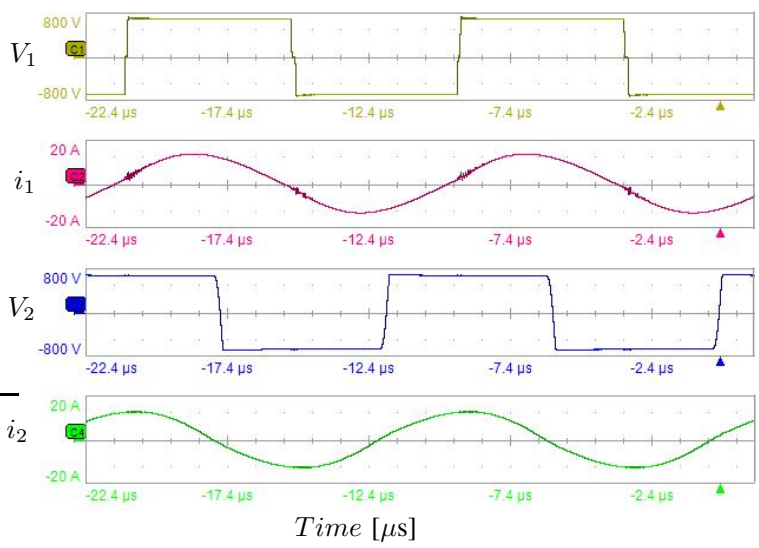

(a) Ferrite pad.

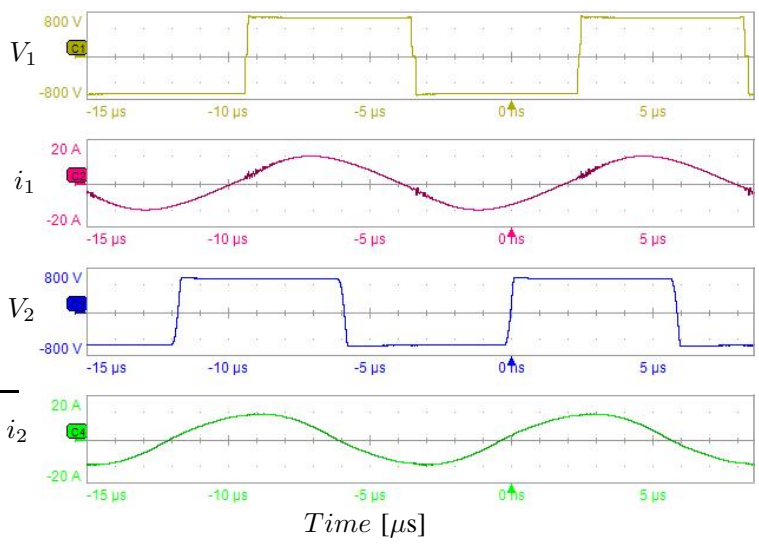

(b) Nanocrystalline pad.

Fig. 11. Current and voltage waveforms at the primary converter, $V_{1}$ and $i_{1}$ as well as at the secondary converter, $V_{2}$ and $i_{2}$. Double-D pad with a) ferrite N87 and $b$ ) nanocrystalline ribbon $t_{c 2}$ cores, respectively. Airgap between pads: $\sim 20 \mathrm{~cm}$. 


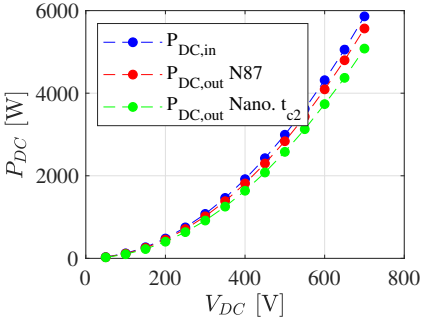

(a)

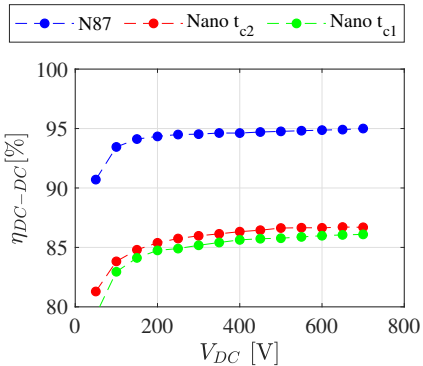

(c)

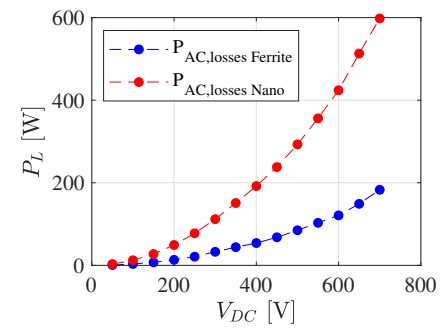

(b)

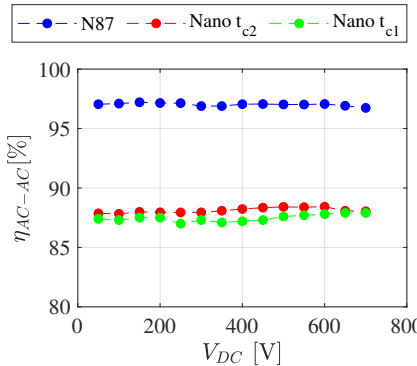

(d)

Fig. 12. Power losses measurement for Double-D pad with nanocrystalline and N87 cores. a) Power DC input. b) Measured power losses in the coils (core and copper losses). c) Overall system efficiency. $d$ ) efficiency of the magnetic coupler (without considering converter losses).

and $97 \%$ for ferrite pads. The power converter efficiency for both cases remains constant at approximately $97.5 \%$ since the operating conditions for the converter are the same. As a result, the overall system efficiency with nanocrystalline

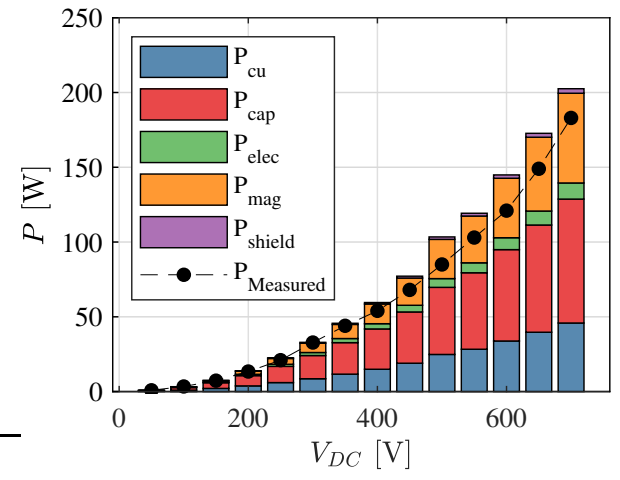

(a) Ferrite

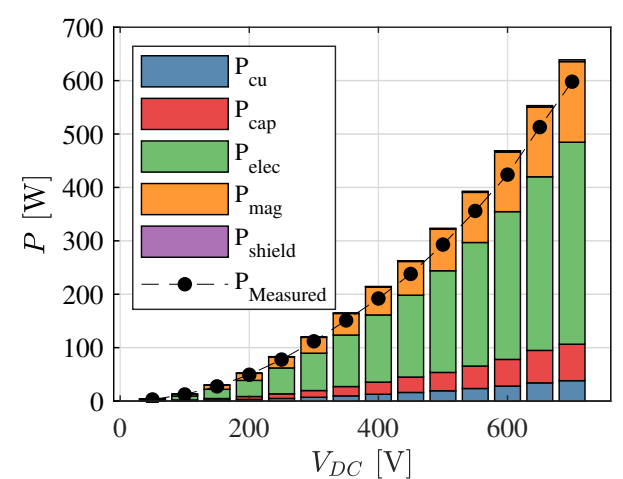

(b) Nanocrystalline $t_{c 2}$
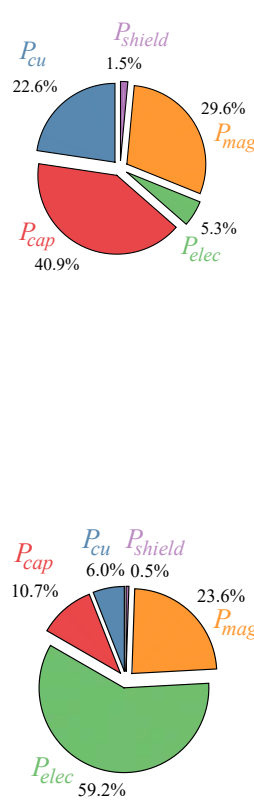
losses (gap losses) from the eddy currents in the lateral faces of the core compromise efficiency. Thus, alternatives for mitigating gap losses are of crucial importance. There are several ways of mitigating these losses. All of these methods could be topics of future work:

1) Optimal core dimensions: In this paper, a one-to-one comparison between nanocrystalline cores is presented; i.e., the ferrite cores are replaced by nanocrystalline cores of identical dimensions. However, better performance of the nanocrystalline pads can be achieved by considering its unique characteristics. higher. Eddy-current loss accounts for almost $60 \%$ of the total loss. On the other hand, the hysteresis loss accounts for $23.6 \%$ of the total loss and it is approximately 2.5 times higher than that of the ferrite pad. All things considered, the of the ferrite cores.

The higher losses of the nanocrystalline pad are mainly a result of the higher conductivity of the core bars and the transverse flux. The $2.5 \%$ increment of the hysteresis losses $P_{m a g}$ is negligible compared to the larger increment of eddycurrent losses $P_{\text {elec }}$. Thus, mitigating the latter is crucial to take advantage of the higher power density achievable by nanocrystalline ribbon cores.

From the analysis presented in this paper, it is clear that nanocrystalline cores can be utilized in IPT pads with the objective of increasing its power density. Nevertheless, the hower losses (gap losses) from the eddy cur
Fig. 13. Breakdown of the calculated losses in the system for different DC-link voltages. $a$ ) ferrite-based Double-D pad. $b$ ) nanocrystalline-based Double-D pad. $P_{c u}$ : Copper losses. $P_{c a p}$ : Capacitor losses. $P_{e l e c}$ : Eddy current losses in the core. $P_{\text {mag }}$ : Hysteresis Losses in the core. $P_{\text {shield }}$ Power losses in the aluminium shield. cores is $88 \%$ and $95 \%$ for ferrite. This efficiency is higher than the minimum efficiency required by the standard SAE J2954 of $85 \%$ but lower than the ferrite counterpart. The nanocrystalline pad with thickness $t_{c 1}$ is about $1 \%$ less effithe one with $t_{c 2}$ despite the fact that more material is used. The lower flux densities are counterbalanced with igher eddy currents which decrease the overall efficiency. Evidently, the reduction of efficiency is the price to pay for ferrite cores; however, for ferrite, the reduction of the core thickness is limited by saturation. Thus, nanocrystalline cores offer an alternative for high power density and high power rating IPT applications. Nevertheless, without further total loss in the pad and segregates it in terms of components. Fig. 13 shows the overall and breakdown losses for ferrite and nanocrystalline pad's where $P_{c u}$ corresponds to the copper loss, $P_{\text {cap }}$ to the capacitor loss, $P_{\text {shield }}$ to the loss in the and hysteresis losses in the core, respectively.

For the ferrite pad, the majority of the loss, $40.9 \%$, corresponds to the compensation capacitors. Magnetic loss accounts for a third of the total loss while the copper loss corresponds to only $22.6 \%$ of the total loss. This is due to the high number of strands used in the coil. On the other for only $1.5 \%$ of the total loss. The difference between the measured and estimated total loss is less than $8 \%$. The estimated total loss closely matches the experimental measurements shown in Fig. 12a).

Similar accuracy has been obtained when estimating the power loss of the nanocrystalline pad. An difference of less than $6 \%$ has been achieved. While the losses in the shield ferrite capacitors are similar to the ones obtained for the 
Fig. 14 compares the magnetic performance as well as the power losses of ferrite-based and nanocrystallinebased cores in terms of percentage of pad's area covered by the cores.

The values of both self and mutual inductances are similar for both materials. The hysteresis power loss $P_{\text {mag }}$ for nanocrystalline cores is slightly higher than that of ferrite cores despite the lower values of Steinmetz coefficients. This is due to the higher localized losses at the lateral faces of the core bars. Nevertheless, the hysteresis power loss decreases with higher pad area coverage due to the reduction of the flux density within the core.

A similar behaviour is observed for eddy current losses. Although almost negligible for ferrite cores, for nanocrystalline cores, they can be up to three times larger than the hysteresis. However, the eddy current losses decrease as the area covered by the core increases. This is due to the fact that less flux enters the lateral faces of the bars, which in turn decreases the induced eddy currents. It is clear that the area coverage has an important impact on the overall losses. The increment of the core material required by larger area coverage can be compensated by a reduction of core thickness, which results in similar or even less core volume.

2) Geometrical modifications to the lateral faces of the cores: In [24], a significant reduction of the gap losses was achieved by sectioning the core. Segmenting the core results in a reduction of the eddy current loop and its associated losses. A similar approach is used in electric machines. The laminations at the edge of the core are sectioned to reduce the eddy current losses as shown in [24]. Following a similar approach, the lateral walls of the nanocrystalline ribbon cores can be segmented with cuts along the $x$ and $y$ axes. The actual reduction of eddy current losses can be analyzed in detail in future work.

3) Special core geometries: Eddy current losses can be also mitigated by reducing the exposure of a planar surface to the traverse flux. Two exemplary cases are shown in Fig.15. In the first one, the laminations at the lateral walls of the cores are placed in a radial manner. In the second one, two longitudinal surfaces of the bar are chamfered into a trapezoidal geometry. In both cases, the exposed planar surface is reduced. The first geometry is convoluted; the second one, however, is more easily realizable. Simulation results of the second

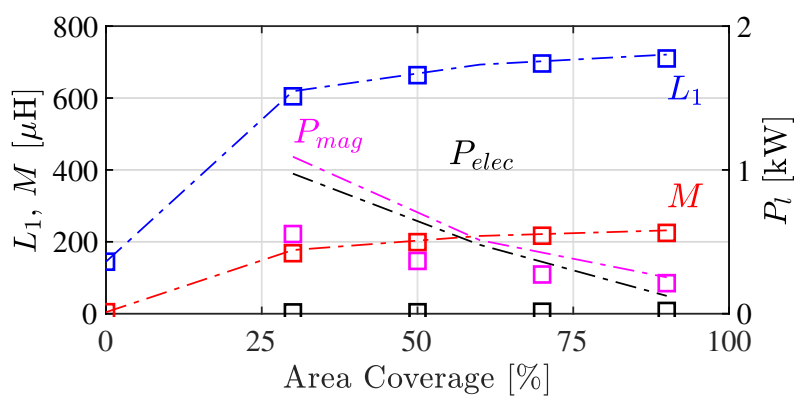

Fig. 14. Self $L_{1}$ and mutual inductance $M$, eddy current losses $P_{\text {elec }}$ and hysteresis losses $P_{m a g}$ for a Double-D pad with percentage of pad's surface covered with core material: $(-.-)$ : Nanocrystalline. $(\square)$ : N87. $t_{c}=16 \mathrm{~mm}$. $i_{1, r m s}=23 \mathrm{~A}$.

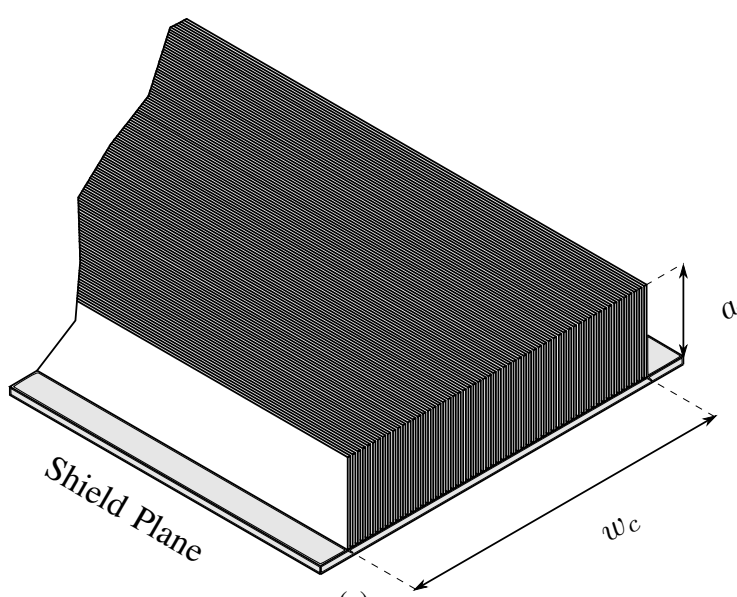

(a)

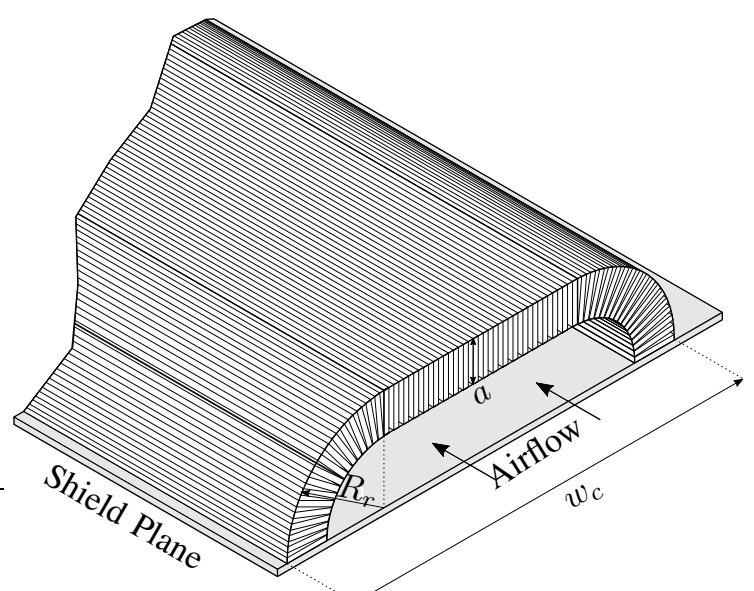

(b)

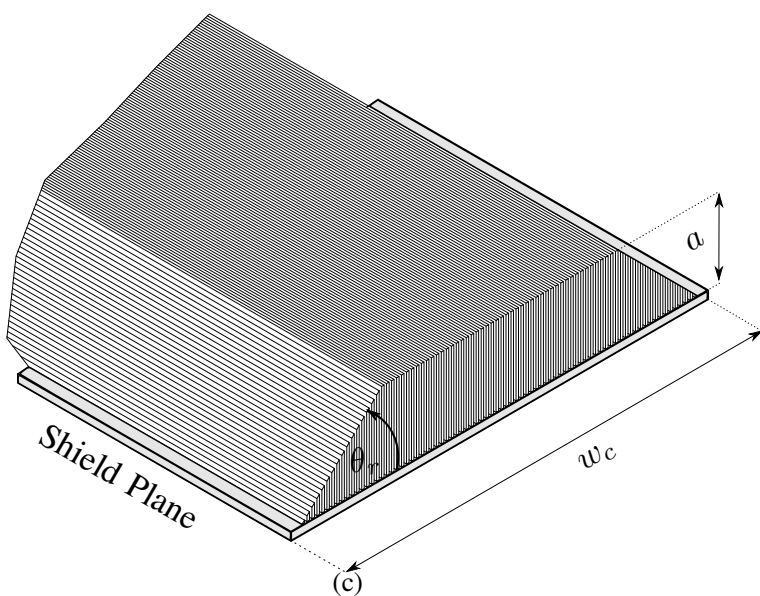

Fig. 15. a) Standard nanocrystalline core. b) and c) Proposed new core geometries to mitigate the induced eddy currents. $w_{c}$ : Core width. $a$ : Ribbon width. $R_{r}$ : radius of curvature.

case suggest a reduction of approximately $54 \%$ of the eddy current losses and $16 \%$ of the hysteresis losses when using this concept as compared to the standard rectangular cores. As future work, an extensive analysis of this concept is required.

4) Hybrid cores: From Fig.8 and [4] it is clear that the flux distribution is not uniform. The mid-section of the core bar withstands higher flux densities as compared 
to the edges of the bar. This allows for a hybrid core that utilizes both ferrite and nanocrystalline ribbon. The higher saturation point of the nano-crystalline bar makes it ideal for the mid-section of the bar. The extremes can still use ferrite. As a result, the higher power density of the pad can still be achieved with a potential reduction of power losses.

\section{CONCLUSIONS}

In this paper, a feasibility analysis of nanocrystalline cores is presented. The main conclusions are:

- The higher permeability of nanocrystalline cores results in a slight improvement of $L, M$ and $k$. However, its high conductivity tends to counterbalance this effect by decreasing $L, M$ and $k$. The overall effect would depend on the core dimensions.

- The flux distribution in the nanocrystalline cores tends to saturate the lateral walls of the core bars. However, the flux density in the majority of the bar is lower than the saturation point, which allows for miniaturization of the magnetic couplers.

- Nanocrystalline core losses are higher than the ones obtained from ferrite cores. The dominant contribution of the loss originates from the eddy-current loss at the core's lateral walls.

- The analytical calculations of power losses closely match the experimental results. This methodology can be used for other ultra-thin laminated cores for IPT applications.

As future work, the optimum placement and geometry of the cores need to be studied. Additionally, new strategies for mitigating the eddy currents losses can be explored.

\section{REFERENCES}

[1] M. Budhia, G. A. Covic, and J. T. Boys, "Design and optimization of circular magnetic structures for lumped inductive power transfer systems," IEEE Transactions on Power Electronics, vol. 26, no. 11, pp. 3096-3108, Nov 2011.

[2] R. Bosshard, U. Iruretagoyena, and J. W. Kolar, "Comprehensive evaluation of rectangular and double-d coil geometry for $50 \mathrm{kw} / 85$ khz ipt system," IEEE Journal of Emerging and Selected Topics in Power Electronics, vol. 4, no. 4, pp. 1406-1415, Dec 2016.

[3] R. Bosshard, "Multi-objective optimization of inductive power transfer systems for ev charging," Ph.D. dissertation, Zurich, 2015.

[4] M. Mohammad and S. Choi, "Optimization of ferrite core to reduce the core loss in double-d pad of wireless charging system for electric vehicles," in 2018 IEEE Applied Power Electronics Conference and Exposition (APEC). IEEE, 2018, pp. 1350-1356.

[5] T. Yilmaz, N. Hasan, R. Zane, and Z. Pantic, "Multi-objective optimization of circular magnetic couplers for wireless power transfer applications," IEEE Transactions on Magnetics, vol. 53, no. 8, pp. 1-12, 2017.

[6] M. Mohammad and S. Choi, "Optimization of ferrite core to reduce the core loss in double-d pad of wireless charging system for electric vehicles," in 2018 IEEE Applied Power Electronics Conference and Exposition (APEC), March 2018, pp. 1350-1356.

[7] T. Yilmaz, N. Hasan, R. Zane, and Z. Pantic, "Multi-objective optimization of circular magnetic couplers for wireless power transfer applications," IEEE Transactions on Magnetics, vol. 53, no. 8, pp. 1-12, Aug 2017.

[8] A. Delgado, G. Salinas, J. Rodrguez, J. A. Oliver, and J. A. Cobos, "Finite element modelling of litz wire conductors and compound magnetic materials based on magnetic nano-particles by means of equivalent homogeneous materials for wireless power transfer system," in 2018 IEEE 19th Workshop on Control and Modeling for Power Electronics (COMPEL), June 2018, pp. 1-5.
[9] H. A. Davies and M. R. Gibbs, Amorphous Alloys. American Cancer Society, 2007. [Online]. Available: https://onlinelibrary.wiley.com/doi/abs/10.1002/9780470022184.hmm401

[10] J. Fzer, S. Dobk, and P. Kollr, "Magnetization dynamics of fecunbsib soft magnetic ribbons and derived powder cores," Journal of Alloys and Compounds, vol. 628, pp. 335 - 342, 2015. [Online]. Available: http://www.sciencedirect.com/science/article/pii/S0925838814030540

[11] J. Füzer, S. Dobák, and P. Kollár, "Magnetization dynamics of fecunbsib soft magnetic ribbons and derived powder cores," Journal of Alloys and Compounds, vol. 628, pp. 335-342, 2015.

[12] J. Fuzerova, J. Fuzer, P. Kollar, L. Hegedus, R. Bures, and M. Faberova, "Analysis of the complex permeability versus frequency of soft magnetic composites consisting of iron andfe ${ }_{73} \mathrm{cu}_{1} \mathrm{nb}_{3} \mathrm{si}_{16} \mathrm{~b}_{7}$, IEEE Transactions on Magnetics, vol. 48, no. 4, pp. 1545-1548, April 2012.

[13] Y. Wang, G. Calderon-Lopez, and A. J. Forsyth, "High-frequency gap losses in nanocrystalline cores," IEEE Transactions on Power Electronics, vol. 32, no. 6, pp. 4683-4690, June 2017.

[14] G. A. C. J.T. Boys, "Ipt fact sheet series: no.1 basic concepts," Deparment of Electrical Engineering. Auckland University, 2011.

[15] R. Bosshard, J. W. Kolar, J. Mhlethaler, I. Stevanovi, B. Wunsch, and F. Canales, "Modeling and $\eta-\alpha$-pareto optimization of inductive power transfer coils for electric vehicles," IEEE Journal of Emerging and Selected Topics in Power Electronics, vol. 3, no. 1, pp. 50-64, March 2015.

[16] S. Kim, A. Tejeda, G. A. Covic, and J. T. Boys, "Analysis of mutually decoupled primary coils for ipt systems for ev charging," in 2016 IEEE Energy Conversion Congress and Exposition (ECCE). IEEE, 2016, pp. 1-6.

[17] A. Bermudez, D. Gomez, and P. Salgado, "Eddy-current losses in laminated cores and the computation of an equivalent conductivity," IEEE Transactions on Magnetics, vol. 44, no. 12, pp. 4730-4738, Dec 2008.

[18] W. H. Hurley, William G.; Wlfle, Transformers and inductors for power electronics. Theory, design and applications, $2 \mathrm{nd}$ ed. WileyBlackwell, 2013.

[19] J. Wang, H. Lin, Y. Huang, and X. Sun, "A new formulation of anisotropic equivalent conductivity in laminations," IEEE Transactions on Magnetics, vol. 47, no. 5, pp. 1378-1381, May 2011.

[20] K. Hollaus, M. Huber, J. Schöberl, and P. Hamberger, "A linear fem benchmark for the homogenization of the eddy currents in laminated media in 3d," IFAC Proceedings Volumes, vol. 45, no. 2, pp. 11901194, 2012.

[21] J. Mühlethaler, "Modeling and multi-objective optimization of inductive power components," Ph.D. dissertation, ETH Zurich, 2012.

[22] M. Budhia, J. T. Boys, G. A. Covic, and C. Y. Huang, "Development of a single-sided flux magnetic coupler for electric vehicle ipt charging systems," IEEE Transactions on Industrial Electronics, vol. 60, no. 1, pp. 318-328, Jan 2013.

[23] R. Bosshard, "Multi-objective optimization of inductive power transfer systems for ev charging," Ph.D. dissertation, ETH Zurich, 2015.

[24] G. Calderon-Lopez, Y. Wang, and A. J. Forsyth, "Mitigation of gap losses in nanocrystalline tape-wound cores," IEEE Transactions on Power Electronics, vol. 34, no. 5, pp. 4656-4664, May 2019.

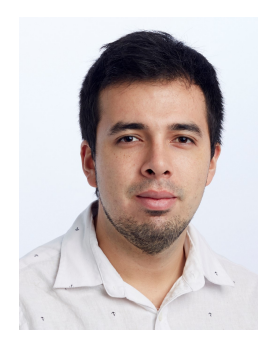

Daniel E. Gaona (SM'14) received the B.S. degree in electrical engineering and the B.S. degree in mechanical engineering from the San Francisco de Quito University, Quito, Ecuador, in 2013 and 2014, respectively. In 2016, he received the M.Sc. degree in electrical engineering from the Erasmus Mundus Master Course (EMMC) Consortium. In 2017, he worked at the Department of Power Electronics and Electrical Drives at the University of Paderborn, Germany, as a Research Associate. In 2018, he joined the University of Cambridge and he is currently working towards his Ph.D. degree. His research interests include electric drives, power electronics, and wireless charging systems. 


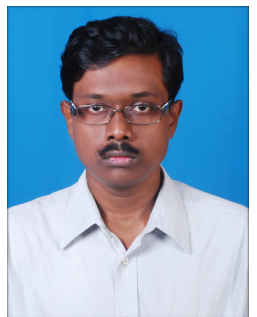

Saikat Ghosh (SM'18) received his BE from Indian Institute of Engineering Science and Technology, Shibpur, India in 2011, and the ME degree in 2013 from the Indian Institute of Science, India. After a two year career as Manager, at Engineering Research Centre of Tata Motors Ltd he joined the University of Cambridge, Engineering Department in 2015 $\mathrm{He}$ currently works towards his Ph.D, and his research interests include electrical drives, particularly for automotive application, power electronics converters and high-frequency converters using wide band gap materials.

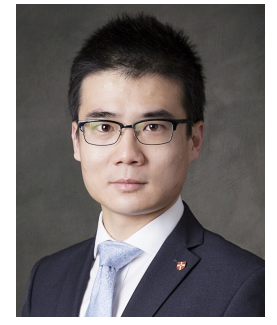

Teng Long (M'13) received the B.Eng. degree from the Huazhong University of Science and Technology, China, the first class B.Eng. (Hons.) degree from the University of Birmingham, UK in 2009, and the Ph.D. degree from the University of Cambridge, UK in 2013. Until 2016, he was a Power Electronics Engineer with the General Electric (GE) Power Conversion business in Rugby, UK. He is currently a Lecturer with the University of Cambridge. His research interests include power electronics, electrical machines, and machine drives. Dr Long is a Chartered Engineer (CEng) registered with the Engineering Council in the UK. 